\title{
Programmed Cell Death in Bacterial Community: Mechanisms of Action, Causes and Consequences
}

\author{
Heejeong Lee and Dong Gun Lee \\ School of Life Sciences, BK 21 Plus KNU Creative BioResearch Group, College of Natural Sciences, Kyungpook National University, Daegu \\ 41566, Republic of Korea
}

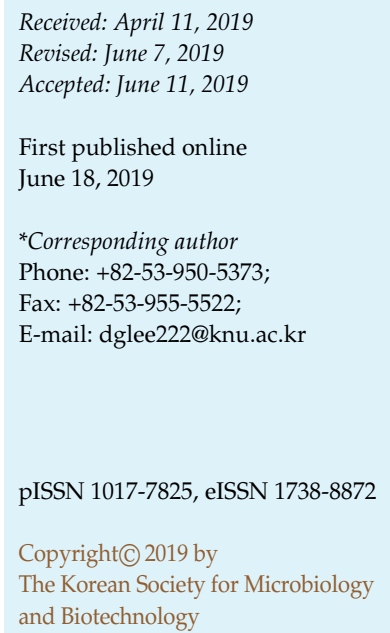

In the bacterial community, unicellular organisms act together as a multicellular being. Bacteria interact within the community and programmed cell death (PCD) in prokaryotes is a sort of altruistic action that enables the whole population to thrive. Genetically, encoded cell death pathways are triggered by DNA damage or nutrient starvation. Given the environmental and bacterial diversity, different PCD mechanisms are operated. Still, their biochemical and physiological aspects remain unrevealed. There are three main pathways; thymineless death, apoptosis-like death, and toxin-antitoxin systems. The discovery of PCD in bacteria has revealed the possibility of developing new antibiotics. In this review, the molecular and physiological characteristics of the three types of PCD and their development potential as antibacterial agents are addressed.

Keywords: Bacterial programmed cell death, apoptosis-like death, thymineless death, mazEF pathway

\section{Introduction}

Programmed cell death (PCD) has been classically investigated in the context of multicellular organisms [1]. PCD in eukaryotes is fundamental for many processes including immunological and stress response, tissue homeostasis, and embryogenesis. For a long period, the importance of PCD had been recognized in multicellular organisms [2]. The intrinsic pathway to apoptosis in eukaryotes is likely to be conserved in prokaryotes, where it provides important functions in response to stress [3]. Over the past two decades, enough evidence has demonstrated the existence of a genetically encoded cell death pathway in single cell organisms in response to environmental stimuli [1, 4]. The PCD system has been reported in various prokaryotes such as Bacillus spp. and Escherichia coli, Anabaena spp., Caulobacter spp., Streptococcus pneumonia, Staphylococcus aureus, Yersinia spp., and Mycobacterium tuberculosis [5]. In optimizing adaptations in the population and development of these organisms, PCD plays critical roles in their survival and pathogenesis, unless they are killed or eaten by predators $[1,6]$. This molecular mechanism releases nutrients from dying cells and supports the growth of the remaining cells in the community [7].

In an antibiotic crisis, novel ways to combat bacterial infections are desperately needed. Although the discovery of self-destruction in the unicellular organism was initially faced with disbelief and criticism, the possibility of developing innovative antibacterial agents that target the artificial activation of these mechanisms is increasingly being recognized [4,8]. These pathways function during biofilm development of $S$. aureus, fruiting body formation of Myxococcus xanthus, and sporulation of Bacillus subtilis [9]. Bacterial PCD also functions as an altruistic mechanism promoting the survival of the bacterial population under stressful conditions such as phage infection and nutrient limitation [9]. Representative prokaryotic PCD pathways described to date are apoptosis-like death (ALD), thymineless death (TLD) and toxin-antitoxin (TA) systems [7]. The physical interaction with its specific target and the complex processes at the biochemical, molecular and ultrastructural 
levels have been investigated [10]. In this review, we describe the mechanisms of PCD from the physiological and molecular perspective.

\section{Advantages of PCD in Bacteria}

The biggest impediment to recognizing the existence of bacterial PCD is the long-held belief that bacteria live solely as unicellular organisms and as such, cellular suicide seems counterintuitive to our understanding of evolutionary processes and the driving forces of natural selection [3]. There is no reason to maintain genes related to the suicide of the free-living individual. However, the genes work on populations, not on individuals [11]. Ranging from insects and mammals to unicellular bacteria, cooperative actions that prevent survival or reproduction exist when the cells lyse, releasing beneficial chemical substances [12]. This behavior appears paradoxical, as it offers no benefit to the individual [13]. Bacterial PCD, as is the case for eukaryotic PCD mechanisms, favors in both the development of multicellular structures and the prolonged preservation of the "organism" as the bacterial population [9]. The induction of bacterial PCD in response to damage might be the last option that occurs only if the cost of repair exceeds the cost of building a new cell [3]. PCD is contributed to the evolutionarily conserved processes for population benefit [7]. Under nutrient starvation, cells release nutrients to increase the fitness of the whole population [10]. Programmed death is often associated with a bacterial stress response, such as DNA deprivation, oxidative stress, and membrane depolarization [13-16]. PCD results in the demand of DNA, RNA, proteins and other cellular essential components for the survival of other starving cells [10]. For instance, biofilms generate nutrients by both releasing nutrients via lysis that can provide nutrition for the remaining population and through decreasing the population density, resulting in increased availability of nutrients per cell [11]. Genotypes that tend to express selfdestructive cooperation can only persist if the phenotype is limited to a part of the object with the genotype, whereas others do not express cooperative behavior and public benefits [12]. Given that a unicellular organism cannot benefit from a PCD, the advantage must be achieved from a larger multicellular structure involving individuals [11]. Moreover, the difference between eukaryotes and bacteria is the release of DNA through PCD. In bacterial cells, the release of DNA can be a goal in itself, and PCD is a means to reach the goal. Due to the release of DNA being inevitable when PCD occurs, free DNA is used for biofilm formation, structural stability and genetic transformation [11].

\section{Apoptosis-Like Death (ALD) Relates to SOS Response}

Apoptosis in eukaryotes commonly occurs to eliminate undesirable cells. One typical and early apoptotic response is phosphatidylserine exposure whereas DNA fragmentation has been associated with a late apoptotic response in eukaryotic cells. Apoptosis in eukaryotic cells shares the main features of ALD, morphologically and mechanistically $[7,11,14]$. The hallmarks of ALD are the following: DNA fragmentation, membrane depolarization, exposure of phosphatidylserine on the cell outer leaflet, rRNA degradation by an endoribonuclease, upregulation of extensive-damage-induced (Edin) genes and high production of hydroxyl radical. Further, the activity of complex I and II of the oxidative respiration system are decreased [7, 11, 17]. Particularly, rRNA degradation implies a relationship between mitochondria-mediated apoptosis and ALD. The mammalian counterpart of the protein-coding gene $\mathrm{YbeY}$ is localized in the mitochondria, hinting at a parallel between mitochondria-mediated apoptosis and ALD [18]. In eukaryotic cells, YbeY has been suggested as playing a role in mitochondria-mediated apoptosis [18]. In bacterial cells, for its part, YbeY is needed for $16 \mathrm{~S}$ rRNA maturation, $70 \mathrm{~S}$ ribosomal assembly, late-stage 70S ribosome quality control, and stress regulation. The protein is recruited to the ribosome where it could cleave the 17S rRNA precursor endonucleolytically at or near the $3^{\prime}$ end maturation site. YbeY is also implicated in the bacterial small RNA regulatory network [19]. In addition, YbeY function is needed for other significant bacterial physiological processes including ALD, transcription antitermination and both Hfq-dependent and Hfq-independent regulation of small RNAs [20]. The loss of function of YbeY, which is involved in mitochondrial mediated apoptosis, leads to the degradation of rRNA. Furthermore, eukaryotes immediately activate DNA repair processes and arrest cell-cycle progression when DNA damage is induced by environmental insult or reactive oxygen species (ROS) accumulation. During repair of the damaged DNA, SulA stops cell division by binding to FtsZ, and this causes filamentation. Cell filamentation is a marker of cell cycle arrest in E. coli that is generally mediated by SulA.

Cell cycle arrest or DNA damage repair response can be evidence of activation of the SOS response [21]. If the amount of cellular DNA damage is overwhelming, 


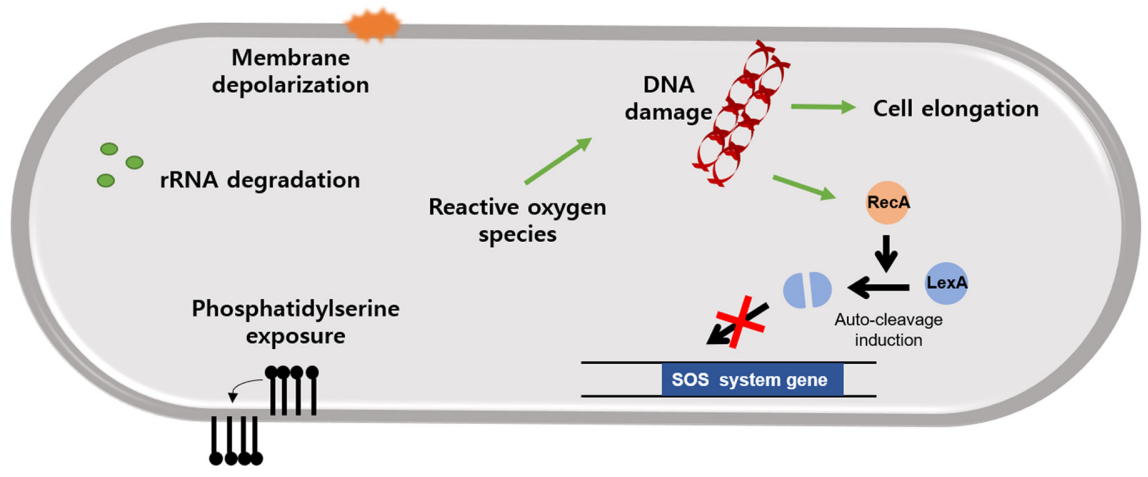

Fig. 1. Overview of apoptosis-like death.

Apoptosis-like death (ALD) is activated by severe DNA damage. ROS are over-produced under ALD conditions. Activation of RecA acts as caspase and occurs ALD. RecA recognized ssDNA and the activated form of RecA stimulates LexA autocleavage. The typical hallmarks are phosphatidylserine externalization, rRNA degradation, cell elongation, membrane depolarization, and DNA fragmentation.

apoptosis is triggered and caspases are deployed to inactivate repair proteins like Rad51, thereby blocking any further attempts to repair the DNA damage and conserving ATP and NADH for programmed cell death [22]. Rad51 family members are homologous to the bacterial RecA protein, which responds to the DNA damage through LexA cleavage and acts on the recruitment of error-prone polymerase in prokaryotes. A composite site for LexA binding and auto-proteolytic cleavage is formed only on the active RecA filament [23]. ssDNA stimulates the conversion of RecA to an active form and the activated RecA promotes autocleavage of the LexA repressor (Fig. 1). They stimulate the SOS response-related genes to repair DNA damage [24-26]. Their operation provides an explanation that answers why some RecA mutants that are not capable of activating the SOS response have increased antibiotic sensitivity [26]. Thus, most of the major bactericidal antibiotics lead to the activation of SOS response, causing the accumulation of toxic hydroxyl radicals [26]. In recent times, the assumption that multifunctional RecA can act like a eukaryotic caspase was reported [14]. RecA has potential as a co-protease function in the bacterial proteolytic system with a caspase substrate binding capacity [22, 23]. In apoptosis, caspase activates various apoptotic factors and other caspases, amplifying the apoptotic signal and leading to cell death. Pleiotropic RecA binds a peptide sequence that serves as a substrate for eukaryotic caspases and antagonistically acts in two forms, like capase-2 [22, 27, 28]. Also, ALD features are not exhibited in $\operatorname{rec} A$ deficient cells $[15,22,28,29]$. It seems that RecA is dependent on the ALD pathway, with a downstream role in the SOS response and due to the damages cannot be improved by the SOS repair system. RecA acts as a patrician in both the SOS response process and ALD pathway, having a slight modification in structure [22] (Fig. 1). Under the condition of severe DNA damage, an extreme SOS response is connected to ALD [18].

\section{Thymine Starvation Proceeds Cell Death by SOS-Dependent or SOS-Independent Pathways}

Thymine is unique among the essential nucleic acid precursors in that it is absolutely required in DNA, but not in RNA. The primary consequence of thymine deprivation involves events at sites where thymine is required as a nucleotide precursor for the initiation of chromosome replication $[30,31]$. When DNA is synthesized, the DNA polymerases can not distinguish thymine from uracil. They only check whether the hydrogen bonds are formed correctly, that is, whether the base pairs are properly matched. Thymine deprivation could lead to ssDNA gaps, causing massive DNA breakage and replication fork stalling [32, 33]. While there is a problem with active replication forks when there is thymine starvation, the primary cause of TLD is that it does not require that the forks be fully activated. TLD still occurs when DNA replication is inhibited [30] and inhibition of transcription by various drugs has relieved TLD [32]. TLD has been confirmed in numerous bacterial species, as well as in other prokaryotes and eukaryotes [30]. Given that TLD is similar in mammalian cells and that certain antibacterial and chemotherapeutic drugs elicit thymine deficiency, a mechanistic understanding of this phenomenon might have valuable biomedical applications [30]. TLD underlies the effectiveness of several antibacterial (trimethoprim, sulfamethoxazole), antimalarial (pyrimethamine, sulfona- 
mide), anticancer (methotrexate, fluorouracil), and immune modulating (methotrexate) agents [31]. Under thymine starvation, the initiation of chromosome replication is a key element in the scenario of TLD [31]. TLD has been associated with DNA recombination structures and DNA damage, as well as their outcomes: induction of suicide modules and prophages, loss of plasmids, mutagenesis, SOS response induction, or filamentation [31]. This death pathway requires several proteins involved in homologous recombination. Cells that accumulate unresolved interchromosomal recombination intermediates die from apparent chromosome-region-specific DNA destruction and chromosome segregation failure [32, 34-36]. The cell death involves both SOS-dependent and SOS-independent pathways: One pathway is dependent on RecA and LexA and requires SOS/SulA induction while another involves homologous recombinational proteins RecQ and RecJ without SOS induction or RecA [32, 37, 38]. RecF loads RecA recombinase onto ssDNA, a precursor to both SOS response and homologous recombination induction. RecF plays a role in the SOS/SulA-dependent pathway, but it also promotes SOS/SulA independently [32]. SulA, which stops the formation of the tubuline-like protein ring and inhibits cell division, cooperates with the SOS-dependent TLD pathway. sulA mutant or SOS-deficient cells may restore growth after starvation has stopped, provided that the sulA did not block cell division [37]. Moreover, the modulation of nucleotide pools by CsrA might inhibit TLD specifically in
SOS- and SulA- deficient cells [34]. After blocking of the cell division through TLD, the cell cycle checkpoint becomes irreversible. Replication of the E. coli chromosome is blocked when undergoing TLD progression. The DNA near the replication origin and terminus are lost. This has been shown to be the result of irreversible cell cycle checkpoints where much of the TLD blocks cell division when the ssDNA accumulates and the rest is the result of DNA breakage [32]. SOS/SulA and the RecA-independent pathway of TLD require RecJ exonuclease and RecQ DNA helicase protein, which together degrade the nascent lagging strands from $5^{\prime}$ ends of the model DNA replication-fork molecules, thereby facilitating fork regression without forming a Holliday junction and exposing ssDNA gaps [30, 32 , 37]. The action of RecJ, having a $5^{\prime}$ to $3^{\prime}$ exonuclease activity on ssDNA, is facilitated by RecQ, which has a $3^{\prime}$ to $5^{\prime}$ helicase activity [39]. Because RecQ helicase can create the $5^{\prime}$-ssDNA-end substrate degraded by RecJ exonuclease, RecJ has a greater resistance to TLD than RecQ. However, RecJ can also degrade 5'-ssDNA ends that arise via means other than RecA [32]. RecQ might catalyze death by recombination in a minor pathway. It has to promote independently of RecA and then by homologous recombination independent mechanism [32].

During thymine starvation, the degradation of the oriC region and initiation events occur. The relationships between TLD and these events have established them to be the main underlying causes of the lethality [31]. DNA

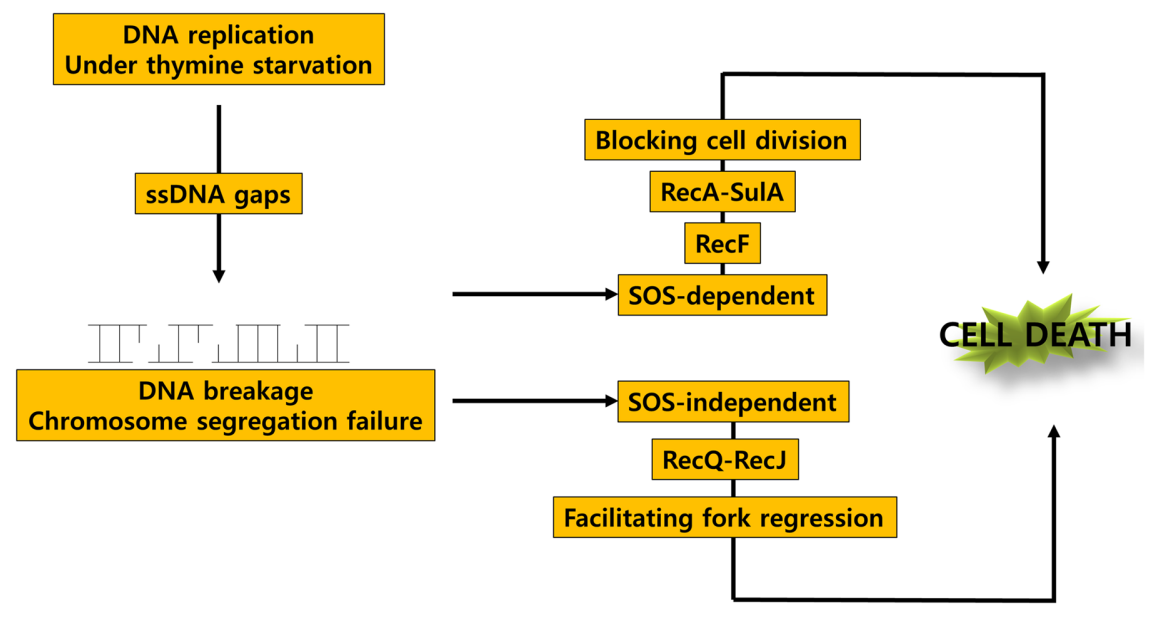

Fig. 2. Overview of thymineless death.

Under thymine starvation condition, attack of dsDNA leads to ssDNA regions and then the initiation of DNA replication or transcription is not required. Extensive DNA breakage results in cell death exacerbating TLD. Induction of SOS pathway relies on RecA-SulA protein. RecA and RecJ protein take charge of SOS-independent pathway. RecF loads RecA recombinase and promotes SulA protein. SulA inhibits cell division. RecJ exonuclease and RecQ helicase degrade the nascent lagging strands. The facilitated replication fork regression occurs without forming a Holliday junction and exposing ssDNA gaps. 
synthesis attempts TLD in the absence of thymine [30]. Because DNA double-stranded breaks are involved in TLD, ssDNA regions/lesions, enlarged by RecQ and RecF, may serve as substrates for DNA breakage as part of a selfdestructive response to stress [35]. Bacterial chromosomes accumulate regions of ssDNA in lagging strands of replication bubbles and at sites of single-stranded lesion repair during TLD. ssDNA lesions are required but insufficient by means of RecFOR or RecQ recombinational repair protein to generate or expand ssDNA regions. Due to the absence of deoxythymidine triphosphate (a source of thymine), these ssDNA region/lesions cannot be filled and persist. Moreover, deficiencies in $\operatorname{rec} F$ and $r e c Q$ protect from TLD [35]. Numerous proteins are associated with control of TLD. The cell death process is exacerbated in cells lacking the YvrD helicase and RecBCD [32]. TLD can also be attenuated by hydroxyl radical and iron scavengers, and by inactivation of $c y d B, u b i G$ and genes that code for respiratory proteins [33]. Also, oppB and $s d h A$ respond to both TLD and ALD pathways but in the opposite manner. Both genes are upregulated during ALD but downregulated in the TLD response [7] (Fig. 2).

\section{mazEF-Mediated Cell Death}

Some bacterial toxins induce inflammation in the host. The pathogens can decrease competitors through manipulation of the host immune system [12]. Some toxins interfere with the essential metabolic processes of the cell and can also lyse the bacterial cell $[12,40]$. The cellular targets of these toxins are protein synthesis, mRNA stability, DNA replication, and synthesis of the cell wall and of ATP [5]. The antitoxins neutralize the toxin through different mechanisms [12, 40] and are mostly responsible for protection against ROS [11]. Antitoxins are required for the survival of a small subset of individuals within the population [11]. Under normal conditions of existence, the antitoxin and toxin are located side by side. They are transcribed and translated together, which limits the lethal effect of the toxin [5]. Toxin-antitoxin (TA) systems, which consist of a bicistronic operon, are widely distributed in the prokaryotic kingdom, often in multiple numbers [40]. There are several pairs of TA modules in E. coli, including mazEF, relBE, chpBIK, prlF-yhaV, yefM-yoeB, and dinJ-yafQ [41]. They are implicated in contradicting phenomena of persistence [40]. TA loci are classified into three types depending on the mode of neutralization of the toxin by the antitoxin $[12,40]$. The antitoxin in the Type I TA system is an antisense RNA that binds to toxin mRNA through complementary base pairing and inhibits the translation of toxins. Type II TA systems are most commonly used with at least eight families based on homology. In this case, the antitoxin directly neutralizes the toxin through proteinprotein interactions [40].

A typical Type II TA system consists of two genes in a single operon. Generally, the toxin-encoding gene is located downstream of the gene encoding the antitoxin under the control of a common autoregulatory promoter. The Type III TA system has recently been discovered and is characterized by RNA-protein interactions, where it binds to the proteic toxins that cause neutralization [40]. The socalled "PCD hypothesis" is represented primarily by the Type II TA system, mazEF module. Many other TA systems, including relBE, have been proven to be bacteriostatic [41]. Toxins are generally globular in structure and have a long half-life. On the other hand, antitoxins are unstable and have a short half-life, because they are degraded by cellular proteolytic enzymes. The dynamic nature of auto-regulation and difference in half-lives of toxins and their cognate antitoxins are vital for the expression and functioning of the TA systems. Under normal conditions, the antitoxin is translated at a higher rate than the toxin to keep the ratio of the antitoxin and toxin constant, neutralizing the toxin continuously [40]. A broad range of causes, such as DNA damage, heat shock, antibiotic stress, nutrient starvation, and other kinds of stresses elicit mazEF-mediated-PCD.

The activation of the mazEF system is achieved through the suppression of transcription or translation of mazEF loci [11]. The MazF toxin is an mRNA interferase, which interferes with protein synthesis. Upon activation, MazF toxin preferentially cleaves tRNA or ssRNA at ACA or $\mathrm{ACU}$ sequences as an endoribonuclease in a subsequent process and ribosome-independent fashion, resulting in cell death. The death proteins are also involved in RNA processing and cleave the MazE antitoxin [11, 42, 43]. The induction of the mazEF-mediated death pathway activates the selective synthesis of two groups of proteins: the products of genes $c l p P, y f i D, s l y D, y f b U$, and $y g c R$, which participate in the death process, and the products of genes $e l a C$ and $d e o C$, which lead to cell survival [18]. The MazFmediated persistence phenotype is independent of RecA and dependent on the presence of $\mathrm{ClpP}$ and Lon protease [42]. mazEF-mediated PCD is controlled by guanosine $3^{\prime}$, $5^{\prime}$-bispyrophosphate (ppGpp) and extracellular death factor (EDF) [11]. ppGpp has been shown to regulate starvationinduced PCD. After identifying the key signaling molecule for amino acid deficiency, the synthesis of ppGpp in E. coli is controlled by two pathways: One is activated by amino 


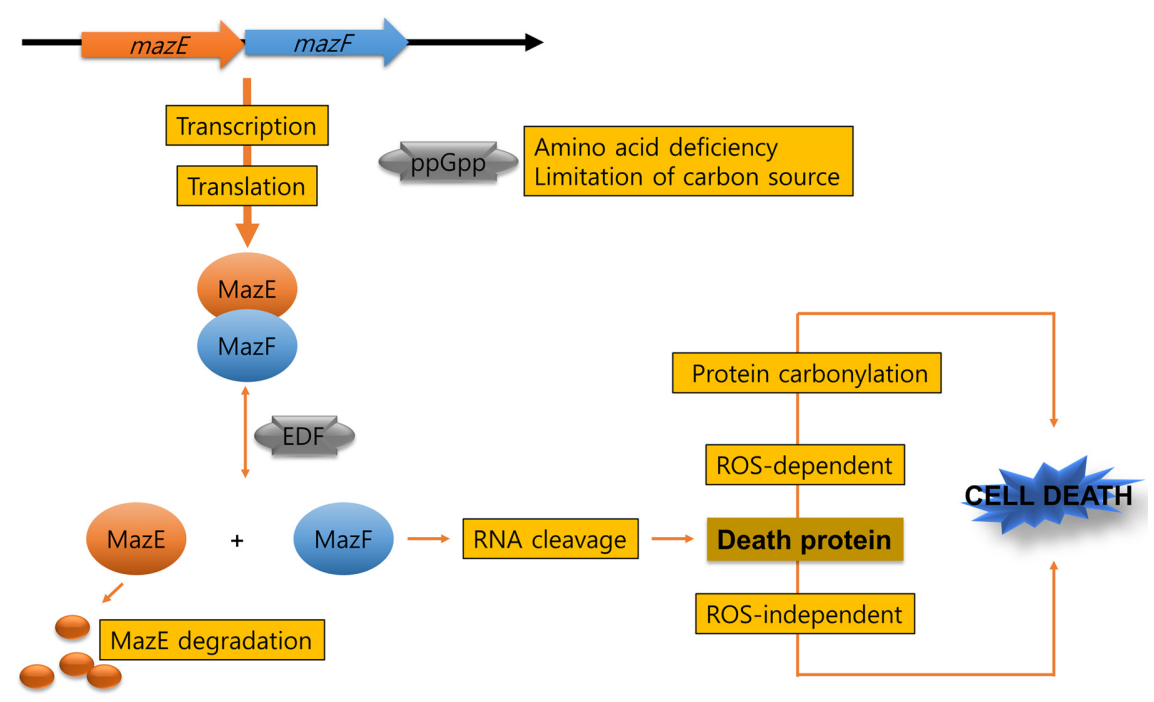

Fig. 3. Overview of programmed cell death by mazEF.

Transcription from promoters leads to the production of MazEF mRNA and translation produces MazE and MazF protein products. Guanosine 3,5 bispyrophosphate (ppGpp) is accumulated under amino acid deprivation or limitation of carbon source. Enhancement of ppGpp reduces transcription mazEF genes. Extracellular death factor is required for the enhancement of mazF activity. Indeed, the EDF- mediated, mazEFmediated pathway would be inactivated. MazE is constitutively degraded by ClpAP protease and therein MazF releases a cleaved RNA form. The mazEF TA system is responsible for nonapoptotic cell death by severe DNA damage. The cell death pathway is divided into ROS-dependent and independent processes.

acid starvation and the enzyme involved in this pathway is encoded by relA, which is either inhibited by amino acylation or induced by uncharged tRNA. The other pathway is activated by the limitation of the relA-independent carbon source. It has been pointed out that an increase of ppGpp in the nutritional deficiency state will inhibit the simultaneous expression of mazE and mazF [41]. A linear pentapeptide quorum sensing signal (NNWNN), EDF, is required for the enhancement of MazF activity [18, 44] and is derived from the modification of glucose-6-phosphate dehydrogenase (G6PD). G6PD is a major metabolic enzyme that catalyzes the first and rate-limiting steps of the pentose phosphate shunting and produces nucleotide precursors. It may be reasonable to deduce that G6PD might also play a significant role in bacterial PCD regulation and that EDF generated by G6PD may link PCD and metabolism [41]. Moreover, depletion of thymine pools was suggested to involve MazF activation in E. coli [45]. Interestingly, mazEFmediated cell death is the opposite of the ALD pathway. The mazEF-mediated death pathway inhibits the recA-lexAdependent ALD pathway in E. coli [18, 46] (Fig. 3).

In conclusion, genetically encoded cell death pathways in bacteria have existed and three types of different PCD mechanisms are described. In light of the evolving antibiotic resistance crisis, novel ways to combat bacterial infections are desperately needed. Innovative strategies are needed to block the development of resistance and to extend the life of antibiotics. The discovery of bacterial PCD provides the possibility of developing a new class of antibacterials targeted at the artificial modulation of PCD in bacteria.

\section{Acknowledgments}

This research was supported by the Basic Science Research Program through the National Research Foundation of Korea (NRF) funded by the Ministry of Science, ICT \& Future Planning (2017R1A2B4005811).

\section{Conflict of Interest}

The authors have no financial conflicts of interest to declare.

\section{References}

1. Tanouchi Y, Lee AJ, Meredith H, You L. 2013. Programmed cell death in bacteria and implications for antibiotic therapy. Trends Microbiol. 21: 265-270. 
2. Allocati N, Masulli M, Di Ilio C, De Laurenzi V. 2015. Die for the community: an overview of programmed cell death in bacteria. Cell Death Dis. 6: e1609.

3. Bayles KW. 2014. Bacterial programmed cell death: making sense of a paradox. Nat. Rev. Microbiol. 12: 63-69.

4. Dewachter L, Verstraeten N, Fauvart M, Michiels J. 2016. The bacterial cell cycle checkpoint protein Obg and its role in programmed cell death. Microb. Cell 3: 255-256.

5. Andryukov BG, Somova LM, Timchenko NF. 2018. Molecular and genetic characteristics of cell death in prokaryotes. Mol. Genet. Microbiol. 33: 73-83.

6. Zheng W, Rasmussen U, Zheng S, Bao X, Chen B, Gao Y, et al. 2013. Multiple modes of cell death discovered in a prokaryotic (cyanobacterial) endosymbiont. PLoS One 8: e66147.

7. Nagamalleswari E, Rao S, Vasu K, Nagaraja V. 2017. Restriction endonuclease triggered bacterial apoptosis as a mechanism for long time survival. Nucleic Acids Res. 45: 8423-8434.

8. Lewis K. 2000. Programmed death in bacteria. Microbiol. Mol. Biol. Rev. 64: 503-514.

9. Dewachter L, Verstraeten N, Monteyne D, Kint CI, Versees W, Perez-Morga D, et al. 2015. A single-amino-acid substitution in Obg activates a new programmed cell death pathway in Escherichia coli. MBio. 6: e01935-01915.

10. Kohanski MA, Dwyer DJ, Collins JJ. 2010. How antibiotics kill bacteria: from targets to networks. Nat. Rev. Microbiol. 8: 423-435.

11. Peeters SH, de Jonge MI. 2018. For the greater good: Programmed cell death in bacterial communities. Microbiol. Res. 207: 161-169.

12. Ackermann M, Stecher B, Freed NE, Songhet $P$, Hardt WD, Doebeli M. 2008. Self-destructive cooperation mediated by phenotypic noise. Nature 454: 987-990.

13. Tanouchi Y, Pai A, Buchler NE, You L. 2012. Programming stress-induced altruistic death in engineered bacteria. Mol. Syst. Biol. 8: 626.

14. Lee W, Lee DG. 2014. Magainin 2 induces bacterial cell death showing apoptotic properties. Curr. Microbiol. 69: 794801.

15. Lee B, Hwang JS, Lee DG. 2019. Induction of apoptosis-like death by periplanetasin-2 in Escherichia coli and contribution of SOS genes. Appl. Microbiol. Biotechnol. 103: 1417-1427.

16. Lee H, Lee DG. 2018. Gold nanoparticles induce a reactive oxygen species-independent apoptotic pathway in Escherichia coli. Colloids Surf. B: Biointerfaces 167: 1-7.

17. Li WR, Xie XB, Shi QS, Zeng HY, Ou-Yang YS, Chen YB. 2010. Antibacterial activity and mechanism of silver nanoparticles on Escherichia coli. Appl. Microbiol. Biotechnol. 85: $1115-1122$.

18. Erental A, Kalderon Z, Saada A, Smith Y, Engelberg-Kulka H. 2014. Apoptosis-like death, an extreme SOS response in Escherichia coli. MBio 5: e01426-01414.
19. Vercruysse M, Köhrer C, Shen Y, Proulx S, Ghosal A, Davies BW, et al. 2016. Identification of YbeY-protein interactions involved in 16S rRNA maturation and stress regulation in Escherichia coli. MBio 7: e01785-01716.

20. Ghosal A, Köhrer C, Babu VM, Yamanaka K, Davies BW, Jacob AI, et al. 2017. C21orf57 is a human homologue of bacterial YbeY proteins. Biochem. Biophys. Res. Commun. 484: 612-617.

21. Lee W, Kim KJ, Lee DG. 2014. A novel mechanism for the antibacterial effect of silver nanoparticles on Escherichia coli. Biometals 27: 1191-1201.

22. Dwyer DJ, Camacho DM, Kohanski MA, Callura JM, Collins JJ. 2012. Antibiotic-induced bacterial cell death exhibits physiological and biochemical hallmarks of apoptosis. Mol. Cell. 46: 561-572.

23. Adikesavan AK, Katsonis P, Marciano DC, Lua R, Herman C, Lichtarge O. 2011. Separation of recombination and SOS response in Escherichia coli RecA suggests LexA interaction sites. PLoS Genet. 7(9): e1002244.

24. Peng Q, Zhou SQ, Yao F, Hou B, Huang YC, Hua DX, et al. 2011. Baicalein suppresses the SOS response system of staphylococcus aureus induced by ciprofloxacin. Cell. Physiol. Biochem. 28: 1045-1050.

25. Kohanski MA, Dwyer DJ, Hayete B, Lawrence CA, Collins JJ. 2007. A common mechanism of cellular death induced by bactericidal antibiotics. Cell 130: 797-810.

26. Steinmann ME, González-Salgado A, Bütikofer P, Mäser P, Sigel E. 2015. A heteromeric potassium channel involved in the modulation of the plasma membrane potential is essential for the survival of African trypanosomes. FASEB J. 29: 3228-3237.

27. Asplund-Samuelsson J. 2015. The art of destruction: revealing the proteolytic capacity of bacterial caspase homologs. Mol. Microbiol. 98: 1-6.

28. Yun DG, Lee DG. 2016. Antibacterial activity of curcumin via apoptosis-like response in Escherichia coli. Appl. Microbiol. Biotechnol. 100: 5505-5514.

29. Lee H, Lee DG. 2019. SOS genes contribute to Bac8c induced apoptosis-like death in Escherichia coli. Biochimie 157: 195-203.

30. Khodursky A, Guzman EC, Hanawalt PC. 2015. Thymineless death lives on: new insights into a classic phenomenon. Annu. Rev. Microbiol. 69: 247-263.

31. Guzman EC, Martin CM. 2015. Thymineless death, at the origin. Front Microbiol. 6: 499.

32. Fonville NC, Bates D, Hastings PJ, Hanawalt PC, Rosenberg SM. 2010. Role of RecA and the SOS response in thymineless death in Escherichia coli. PLoS Genet. 6: e1000865.

33. Matic I. 2018. The major contribution of the DNA damagetriggered reactive oxygen species production to cell death: implications for antimicrobial and cancer therapy. Curr. Genet. 64: 567-569. 
34. Hamilton HM, Wilson R, Blythe M, Nehring RB, Fonville NC, Louis EJ, et al. 2013. Thymineless death is inhibited by CsrA in Escherichia coli lacking the SOS response. DNA Repair (Amst). 12: 993-999.

35. Hong Y, Li L, Luan G, Drlica K, Zhao X. 2017. Contribution of reactive oxygen species to thymineless death in Escherichia coli. Nat. Microbiol. 2: 1667-1675.

36. Khan SR, Kuzminov A. 2019. Thymineless death in Escherichia coli is unaffected by the chromosomal replication complexity. J. Bacteriol. 00797-00718.

37. Hastings PJ, Rosenberg SM. 2017. A radical way to die. Nat. Microbiol. 2: 1582-1583.

38. Fonville NC, Vaksman Z, DeNapoli J, Hastings PJ, Rosenberg SM. 2011. Pathways of resistance to thymineless death in Escherichia coli and the function of UvrD. Genetics 189: 23-36.

39. Morimatsu K, Kowalczykowski SC. 2014. RecQ helicase and RecJ nuclease provide complementary functions to resect DNA for homologous recombination. Proc. Natl. Acad. Sci. USA 111: E5133-5142.

40. Ramisetty BC, Natarajan B, Santhosh RS. 2015. mazEFmediated programmed cell death in bacteria: "what is this?". Crit. Rev. Microbiol. 41: 89-100.
41. Hu MX, Zhang X, Li EL, Feng YJ. 2010. Recent advancements in toxin and antitoxin systems involved in bacterial programmed cell death. Int. J. Microbiol. 2010: 781430.

42. Tripathi A, Dewan PC, Siddique SA, Varadarajan R. 2014. MazF-induced growth inhibition and persister generation in Escherichia coli. J. Biol. Chem. 289: 4191-4205.

43. Schifano JM, Cruz JW, Vvedenskaya IO, Edifor R, Ouyang M, Husson RN, et al. 2016. tRNA is a new target for cleavage by a MazF toxin. Nucleic Acids Res. 44: 1256-1270.

44. Kolodkin-Gal I, Hazan R, Gaathon A, Carmeli S, EngelbergKulka H. 2007. A linear pentapeptide is a quorum-sensing factor required for mazEF-mediated cell death in Escherichia coli. Science 318: 652-655.

45. Davies BW, Kohanski MA, Simmons LA, Winkler JA, Collins JJ, Walker GC. 2009. Hydroxyurea induces hydroxyl radical-mediated cell death in Escherichia coli. Mol. Cell 36: 845-860.

46. Erental A, Sharon I, Engelberg-Kulka H. 2012. Two programmed cell death systems in Escherichia coli: an apoptotic-like death is inhibited by the mazEF-mediated death pathway. PLoS Biol. 10(3): e1001281. 